\title{
Electrooptical behavior of twisted-wedge nematic structures
}

\author{
J. A. Martin-Pareda, M. A. Muriel, and J. M. Oton
}

\begin{abstract}
A new type of domain in twisted-wedge negative nematics is reported. The wedge shape allows the separation of ordinary and extraordinary beams that can be studied separately. In these conditions, at least five different regions can be detected, depending on applied voltages and frequencies. Both rays are shown to yield different diffraction patterns. The relative light intensity of several spots of these patterns is also studied.
\end{abstract}

\section{Introduction}

In the last few years, increasing interest in the use of liquid crystals (LC) as nonlinear optical media has developed. A large number of papers ${ }^{1}$ have been devoted to this field. Several LC-based optical devices using different configurations and electric, magnetic, or optical $^{2-12}$ external fields have been proposed. Moreover LC cells have offered a new interest in the field of optical bistability. Certain new effects, closely related to the path to chaos, have been found. These phenomena open interesting possibilities in a field where many new findings are still appearing.

On the other hand, although electrohydrodynamic instabilities in negative nematic LCs have been extensively studied since the pioneering works by Freedericksz ${ }^{13}$ and Naggiar, ${ }^{14}$ there are many phenomena, not reported yet, that should be taken into consideration for a better understanding of the dynamic behavior of these materials.

In this paper, a new kind of electrooptical effect, based on one reported by some of us, ${ }^{15}$ is presented. The main interest in this effect is its unique electrohydrodynamic behavior. In some cases, this effect could be employed in light modulation in the same way as in the laser beam modulator reported previously. ${ }^{12}$ Our present objective is to show that different domains can be obtained in twisted-wedge nematic structures and, at the same time, study their time response when a pulsed signal is applied to the LC cell. As will be shown, new types of domain, other than Williams and chevron commonly reported, take place for different ranges of voltages and frequencies.

Ogawa et al. ${ }^{16}$ described an electrooptical effect in a twisted-nematic liquid cell. Their experiment shows a twisted-nematic structure induced by an electric field. The initial configuration was a homeotropic one, but a previous rubbing of the glass plates imposes its action

The authors are with Universidad Politecnica de Madrid, ETSI Telecomunicacion, Ciudad Universitaria, Madrid-3, Spain.

Received 20 January 1983.

0003-6935/84/132159-04\$02.00/0.

(C) 1984 Optical Society of America. when a certain electric field is applied. If the rubbing direction for the glass plate at one side of the liquid crystal layer is perpendicular to the opposite one, the resulting Williams domain pattern is formed at $45^{\circ}$ with respect to both directions. No indications of the effect of polarization of the incident radiation on the output beam polarization are given. The transmitted-light response curves, measured with $\mathrm{He}-\mathrm{Ne}$ laser light and parallel polarizers, are shown for two frequencies, namely, 10 and $100 \mathrm{kHz}$. Another experiment of this type has been reported by Raynes, ${ }^{17}$ but in this case a twisted configuration is used from the beginning. Williams domains are again reported, and the possibility of obtaining the molecular rotation direction depending on the Williams domain direction is shown.

Both experiments are done with planar structures and with polarized light. Hence no results are given for unpolarized laser light. This is the usual experimental setup when dealing with planar cells, since ordinary and extraordinary rays move through the same path. However, wedge structures make possible separating both beams, yielding new effects depending upon the beam chosen for the experiment.

\section{Experimental}

$N$ - ( $p$ - methoxybenzylidene) - $p$ - $n$ - butylaniline (MBBA) synthesized by us ${ }^{18}$ has been used as a nematic LC. The working temperature is $25^{\circ} \mathrm{C}$. Glass plates coated with transparent $\mathrm{SnO}_{2}$ electrodes deposited by conventional spraying are used.

The device (Fig. 1) is a wedge structure constructed with two glass plates separated by a $125-\mu \mathrm{m}$ Mylar spacer placed at one edge of the cell; a prismatic angle of $\sim 1^{\circ}$ is obtained in this way. The molecular configuration is a $\pi / 2$ twist obtained by the classical mechanical and chemical surface pretreatment described elsewhere. ${ }^{19}$ A similar device with a different molecular configuration has been proposed by us ${ }^{4,5}$ as an analog and digital light deflector as well as an optical modulator.

Square-wave voltage signals of up to 50-V P-P were imposed with frequencies from $1 \mathrm{~Hz}$ to $10 \mathrm{kHz}$.

The diffraction patterns (Fig. 2) were observed by 


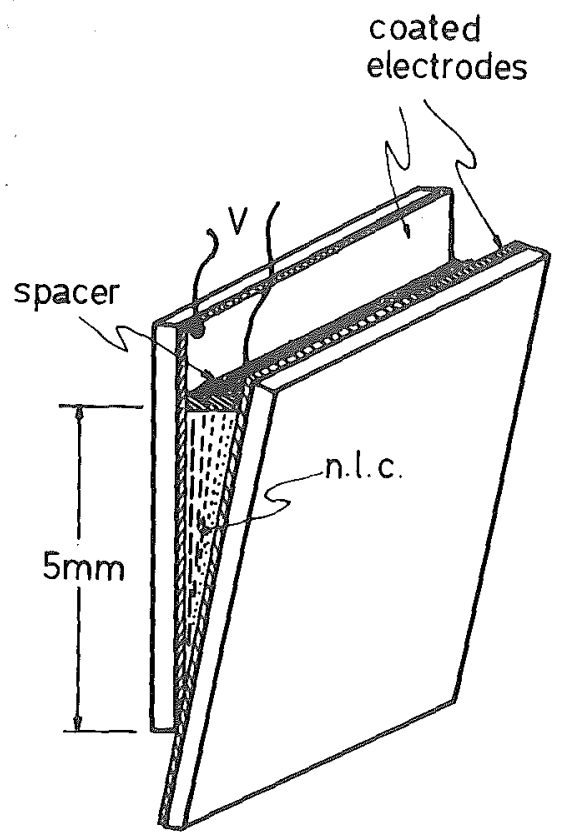

Fig. 1. Wedge-shaped structure used for the experiments.

projecting from the sample onto a screen located at 1.5 $\mathrm{m}$. Polarized and unpolarized $0.5-\mathrm{mW} \mathrm{He}-\mathrm{Ne}$ laser beams were employed in the study. The incident beam was in all cases orthogonal to the frontal surface of the cell. The laser beam diameter on the cell was $0.4 \mathrm{~mm}$. The thickness of the region under study was $\sim 30 \mu \mathrm{m}$. Polarization of the output light was studied by a polarizing beam splitter.

Light intensities were measured at different points of the pattern by a TIL 81 phototransistor working in the photodiode mode located far enough $(1.5 \mathrm{~m})$ from the cell.

\section{Resulis}

With the scheme described above and no voltage imposed, either one or two spots appear on the screen depending on the laser polarization. When a nonpolarized laser is used, the prismatic structure splits the beam into its ordinary and extraordinary components. ${ }^{20}$

When a certain voltage is applied, the above situation starts to change depending on the applied voltage and the frequency. To achieve a better understanding of the results, two different experimental configurations (Fig. 3) have been employed. In Fig. 3(a), the LC molecules are arranged so that the refractive index seen by a polarized laser beam is the extraordinary one. The output light will be, according to the twisted configuration, orthogonally polarized with respect to the input beam. In Fig. 3(b) the refractive index seen by the light is the ordinary one. As in the above case, the emerging laser ray will show a polarization orthogonal to the incident beam. The resulting effects are different depending on the chosen configuration.

In a recent paper ${ }^{15}$ it was shown that four different regions-no domains, Williams, Sigilliaria, and dynamic scattering - could be formed in twisted structures by raising the $\mathrm{P}-\mathrm{P}$ amplitude of a square ac voltage at fixed

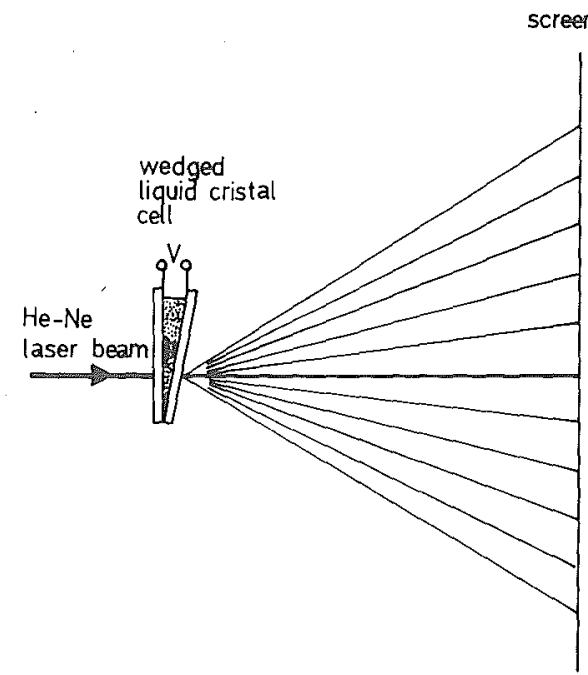

Fig. 2. Experimental setup.

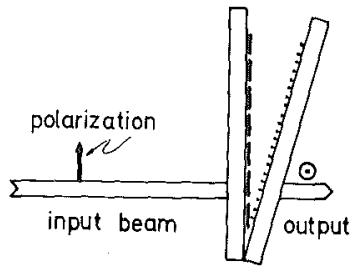

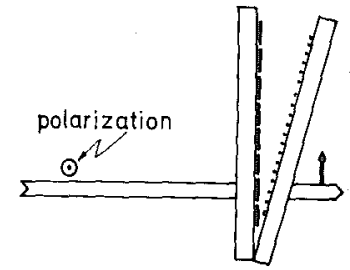

Fig. 3. Experimental arrangement for polarized beams. The incident beam acts as an extraordinary (a) or ordinary (b) beam; the output beam at $0 \mathrm{~V}$ is always perpendicular to the input beam.

frequency between 1 and $1000 \mathrm{~Hz}$. In that paper, an unpolarized laser beam was taken as the input beam, and no distinction was made between both types of polarization, that is, normal or parallel to the direction of the first molecular layer. A closer look, taking into account these considerations, allows detection of a fifth region in the same frequency range.

If we use the arrangement shown in Fig. 3(a), the following steps are obtained:

$$
\text { no domains } \stackrel{11-V_{P-P}}{\longrightarrow} \text { fish-bone domains } \stackrel{12-V_{P-P}}{\longrightarrow} \text { scattering, }
$$

where fish-bone is the tentative name of the diffraction pattern (Fig. 4) now found. The texture domains of this pattern are shown in Fig. 5. The numbers over the arrows are typical values of peak-to-peak voltages in the experiment. The conditions were: $30-\mu \mathrm{m}$ sample thickness; $25^{\circ} \mathrm{C}$ temperature, $100-\mathrm{Hz}$ fixed frequency. These voltages are slightly affected by thickness and temperature but are almost constant with frequency between 10 and $500 \mathrm{~Hz}$.

An interesting feature of these domains is that the size of the pattern, as projected onto the screen, is strongly dependent on the voltage and frequency. As a matter of fact, the pattern becomes larger as the voltage increases and smaller when the frequency is raised. Both formation and variation of the pattern may take a fairly long time, 1-2 min and even more, depending on the working temperature. 


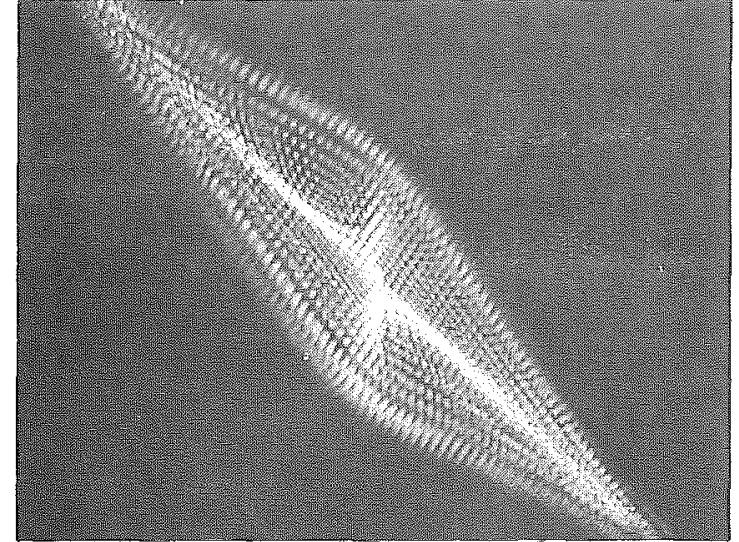

Fig. 4. Fish-bone diffraction pattern. This picture was taken at $11.2-\mathrm{V}_{\mathrm{P}-\mathrm{P}}, 100 \mathrm{~Hz}$.

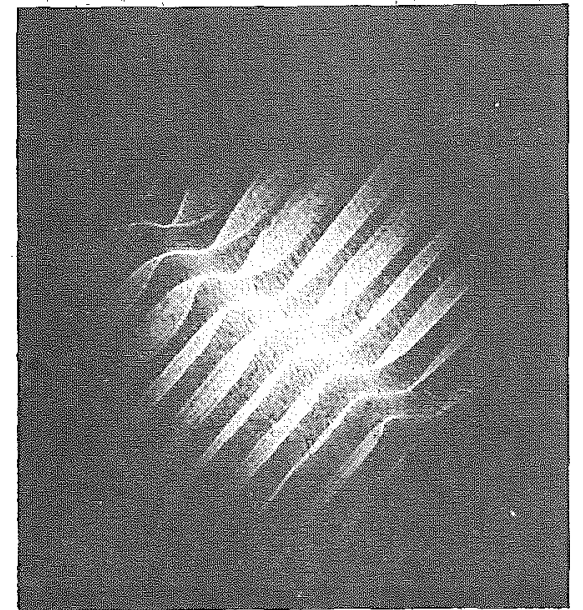

Fig. 5. Texture domains yielding the diffraction pattern of Fig. 4.

Once the pattern is formed at a fixed voltage and a frequency below $10 \mathrm{~Hz}$, an oscillation of its size can be observed. Above this range the pattern becomes static for every frequency, probably because the response time of the LC is too large. On the other hand, the arrangement shown in Fig. 3(b) gives the following steps:

no domains $\stackrel{12-\mathrm{V}_{\mathrm{P} P \mathrm{P}}}{\longrightarrow}$ Williams $\stackrel{20 \mathrm{~V}}{\longrightarrow}$ Sigilliaria $\stackrel{32 \mathrm{~V}}{\longrightarrow}$ dynamic scattering.

Again the numbers over the arrows are peak-to-peak voltages. Experimental conditions remain the same. As before, these voltages are affected by thickness and temperature but are almost constant with frequency up to $0.5 \mathrm{kHz}$ depending on the pattern. Both Williams ${ }^{21}$ and Sigilliaria ${ }^{15}$ domains have been reported previously, so there are no pictures of them here.

The combined result of these two experiments can be shown by using an unpolarized laser input:

$$
\begin{gathered}
\text { no domains } \stackrel{11 \mathrm{~V}}{\longrightarrow} \text { fish-bone } \stackrel{12 \mathrm{~V}}{\longrightarrow} \text { Williams } \stackrel{20 \mathrm{~V}}{\longrightarrow} \\
\text { Sigilliaria } \stackrel{32 \mathrm{~V}}{\longrightarrow} \text { dynamic scattering. }
\end{gathered}
$$

Here we start with two (ordinary and extraordinary)

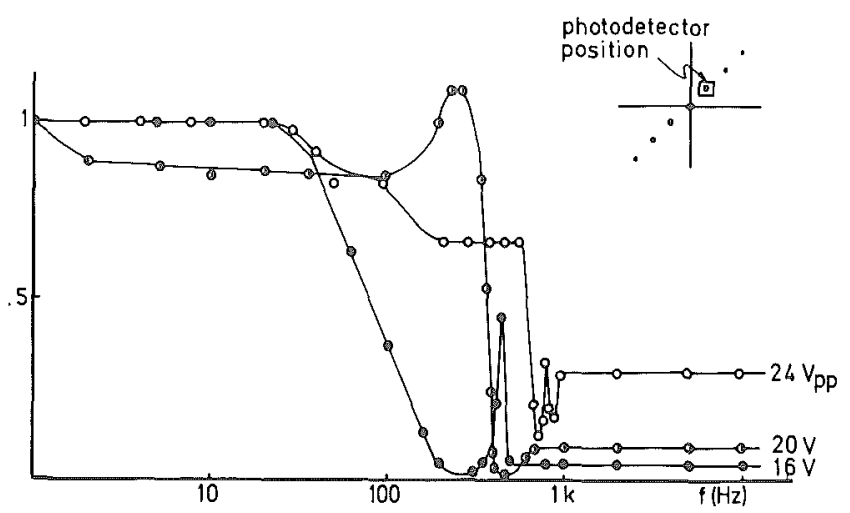

Fig. 6. Relative light intensity striking the first spot (see inset) of Williams or Sigilliaria domains at different frequencies for three voltages: $24-V_{P-P}(0) ; 20-V_{P-P}(\oplus)$; and $16-V_{P-P}(\odot)$. See text for details.

spots in the screen. When voltage is raised to $11-V_{P-P}$, fish-bones are formed about the extraordinary beam position, whereas the ordinary spot is unchanged. At $12-V_{P-P}$ Williams domains are formed about the ordinary beam spot position, polarized as the ordinary beam; extraordinary polarized light is scattered out. From this point, the pattern formed (i.e., Williams and Sigilliaria) are polarized like the ordinary beam.

Two points should be made concerning the above sequences: First, the extraordinary ray is scattered out at 12 -V P-P, whereas the ordinary one remains unaffected or forms a stable pattern. Therefore the $12-\mathrm{V}$ scattering cannot be considered a classical 3-D dynamic scattering. Second, the Williams domains are to be taken as domains whose diffraction pattern as well as texture resembles the well-known Williams domains, the only difference being that they are generated by ordinary beams.

To achieve a better understanding of the above phenomena, some measurements have been taken using the unpolarized laser beam. Although the patterns themselves do not depend on the frequency, the relative intensity of the spots does. Figure 6 is a plot of light intensity vs frequency for several applied voltages normalized at $f=1 \mathrm{~Hz}$. This has been done by selecting one of the two spots closest to a central ordinary beam spot (see inset of the same figure) in the Williams pattern and the equivalent position of Sigilliaria pattern.

Several features appear in this plot. Up to $8-15 \mathrm{~Hz}$, depending on the sample thickness, the intensity is modulated at the same frequency as the voltage. In these cases, the mean value has been represented. Above this range, the pattern becomes static. The light intensity in Fig. 6 decreases as the frequency increases, the decrease being more abrupt for higher voltages. These curves should be considered together with the ones shown in our previous paper, ${ }^{15}$ where a plot of voltage vs frequency was represented showing the regions where the different patterns are formed. It can be seen there that a reverse pathway (Sigilliaria $\rightarrow$ Williams) can be obtained by raising the frequency for a certain constant voltage. The $16-V_{P-P}$ curve of Fig. 6 shows the transition Williams $\rightarrow$ fish-bone. Indeed, 


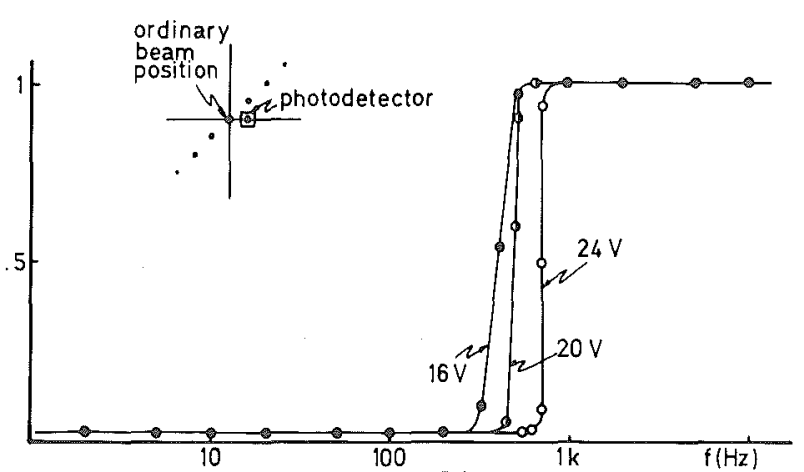

Fig. 7. Relative light intensity striking the zero-voltage screen position of the extraordinary beam (see inset). Symbols as in Fig. 6 .

below $100 \mathrm{~Hz}$ the sample is well within the region in which Williams domains are formed. When the frequency is raised, the light intensity decreases, but a sharp peak can be detected at $440 \mathrm{~Hz}$ corresponding to the formation of the fish-bone pattern, which is close enough to the point the light is being monitored to give some residual light on the position of the previous Williams spot. If the frequency is raised again, the fish-bone pattern collapses to yield the original extraordinary beam spot.

The same steps are shown in Fig. 7 where the light detector (see inset) has been placed in the position of the extraordinary spot when no voltage is applied. The curves $20-\mathrm{V}$ and $24-\mathrm{V}_{\mathrm{P}-\mathrm{P}}$ correspond in a general way to the decreases of their homologue in Fig. 6. The $16-V_{P-P}$ curve, however, starts rising when the fish-bone pattern is formed and keeps rising when it collapses as the extraordinary spot is regenerated.

Some preliminary experiments have been performed on planar-twisted and wedge-homogeneous samples, trying to elucidate the origin of the above textures. Planar-twisted structures merely yield Williams domains as described. ${ }^{17}$ However, wedge-homogeneous cells show a more complex dynamics; indeed some other textures besides Williams domains become apparent for certain voltage-frequency regions. These textures are also different from the above described. On the other hand, only the extraordinary beam is apparently active in this case.

\section{Conclusions}

Several new facts have been reported in this paper. The first is the existence of a new type of domain not reported previously. Moreover, although this domain could be considered as a variation of Williams domains, it offers the difference of being obtained from a different ray polarization. On the other hand, the pattern size depends on the applied voltage and frequency.

Wedge-structure behavior depends on the polarization of the incident beam; the resulting domains are different when dealing with ordinary and extraordinary beams.

These structures show the peculiar characteristic of forming Williamslike domains with ordinary beams. To the best of our knowledge, this is the first time such a feature has been reported.
A more detailed comparison of the above results with those obtained with other cell types, namely, wedgeparallel and planar-twisted aligned cells, is under study by us.

\section{References}

1. See, for example, H. Kelker and R. Hatz, Handbook of Liquid Crystals (Verlag Chemie, Weinheim, 1980), pp. 194-202, where more than 30 references are given.

2. I. C. Khoo and S. L. Zhuang, "Nonlinear Optical Amplification in a Nematic Liquid Crystal Above the Frederiks Transition," Appl, Phys. Lett. 37, 3 (1980).

3. I. C. Khoo, S. L. Zhuang, and S. Shepard, "Self-Focusing of a Low Power cw Laser Beam via Optically Induced Birefringence in a Nematic Liquid Crystal Film," Appl. Phys. Lett. 39, 937 (1981).

4. M. A. Muriel and J. A. Martin-Pereda, "Analog Light Beam Deflector with Liquid Crystals," J. Opt. Soc. Am. 70, 1610A (1980).

5. J. A. Martin-Pereda and M. A. Muriel, "Digital Light Beam Deflector with Liquid Crystals," Europhys. Conf. Abstr. 4I, L2 (1980).

6. J. A. Martin-Pereda and M. A. Muriel, "Magneto-Optical Deflector with Liquid Crystals," Proc. Tech. Program. Electroopt./Laser 80, 199 (1980).

7. S. D. Durbin, A. M. Arakelian, and Y. R. Shen, "Optical-FieldInduced Birefringence and Freedericksz Transition in a Nematic Liquid Crystal," Phys. Rev. Lett. 47, 1411 (1981).

8. A. S. Zolotko, V. F. Kitaeva, N. Kroo, N. N. Sobolev, and L. Chillag, "The Effect of an Optical Field on the Nematic Phase of the Liquid Crystal OCBP," JETP Lett. 32, 158 (1980).

9. R. M. Herman and R. J. Sherinko, "Nonlinear-Optical Processes in Nematic Liquid Crystals near Freedericksz Transition," Phys. Rev. A 19, 1757 (1979).

10. B. Ya. Zeldovich, N. F. Pilipetskii, A. V. Sukhov, and N. V. Tabiryan, "Giant Optical Nonlinearity in the Mesophase of a Nematic Liquid Crystal (NCL)," JETP Lett. 31, 263 (1980).

11. J. A. Martin-Pereda and F. J. López, "Opto-optical Modulation in $N$-( $p$-methoxybenzylidene)-p-butylaniline," Opt. Lett. 7, 590 (1982).

12. J. A. Martin-Pereda and M. A. Muriel, "Liquid-Crystal Electro-optic Modulator Based on Electrohydrodynamic Effects," Opt. Lett. 5, 494 (1980).

13. V. Fréedericksz and V. Tsvetkov, "Bewegung Anisotroper Flüssigkeiten im Elektrischen Feld," Acta Physicochim. URSS 3, 879 (1935).

14. V. Naggiar, "Production des fils et des tourbillons dans les liquides nematiques," C.R. Acad. Sci. 200, 903 (1935).

15. J. A. Martín-Pereda and M. A. Muriel, "Electrohydrodynamic Behavior in Twisted-Wedge Nematic Structures," Mol. Cryst. Liq. Cryst. 98, 183 (1983).

16. F. Ogawa, C. Tani, and F. Saito, "New Electro-Optical Effect: Optical Activity of Electric-Field-Induced Twisted-Nematic Liquid Crystals," Electron Lett. 12, 70 (1976).

17. E. P. Raynes, "Twisted Nematic Liquid-Crystal Electro-Optic Devices with Areas of Reverse Twist," Electron. Lett. 9, 101 (1973).

18. P. Keller and Liebert, "Liquid Crystals for Physicists," in Liquid Crystals, L. Liebert, Ed. (Academic, New York, 1978).

19. E. L. Williams, Liquid Crystals for Electronic Devices (Noyes Data Corp., N.J., 1975), pp. 193-204.

20. S. Sato and A. Kikuchi, "Light Deflection by Nematic Liquid Crystal Cells," Oyo Butsuri 45, 938 (1976).

21. R. Williams, "Domains in Liquid Crystals," J. Chem. Phys. 39, 384 (1963). 